International Mathematical Forum, 1, no. 20, 2006, $973-1000$

\title{
Fuzzy-Identification-Based Adaptive Sliding-Mode Control for a Class of Nonlinear Systems
}

\author{
Chun-Fei Hsu* \\ Department of Electrical and Control Engineering, \\ National Chiao-Tung University, \\ Hsinchu, 300, Taiwan, Republic of China \\ E-mail: fei@cn.nctu.edu.tw \\ Chih-Min Lin \\ Department of Electrical Engineering, Yuan-Ze University, \\ Chung-Li, Tao-Yuan, 320, Taiwan, Republic of China \\ E-mail:cml@staurn.yzu.edu.tw \\ Chin-Hsu Leng \\ Department of Electrical Engineering, Yuan-Ze University, \\ Chung-Li, Tao-Yuan, 320, Taiwan, Republic of China \\ E-mail: s939102@mail.yzu.edu.tw
}

\begin{abstract}
In this study, a fuzzy-identification-based adaptive sliding-mode control (FIASMC) scheme is proposed for a class of nonlinear systems. The FIASMC is comprised of a computed controller and a robust controller. The computed controller containing a fuzzy identifier is the principal controller, and the robust controller is designed to dispel the identification error. In the FIASMC, the parameter adaptation laws are derived in the sense of Lyapunov, so that the stability of the system can be guaranteed. To investigate the effectiveness of the FIASMC, it is applied to control a chaotic Duffing system. The simulation results demonstrate that the proposed FIASMC system can achieve favorable control performance.
\end{abstract}

Keywords: adaptive control, fuzzy control, sliding-mode control, nonlinear systems, chaotic system

* Corresponding Author 


\section{Introduction}

Most current techniques for designing control systems are based on a good understanding of the plant under consideration and its environment. However, in a number of instances, the plant to be controlled is too complex and the basic physical processes in it are not fully understood. When the exact model of controlled system is well known, there exists an ideal controller to achieve satisfactory control performance by possible canceling all the system uncertainties. Since the system parameters and the external disturbances may be perturbed or unknown, the ideal controller is always unimplementable. Assume that all uncertainties existed in the controlled system are bounded, a robust design technique referred to as sliding-mode control has been presented to confront these uncertainties [1]. However, to satisfy the robust stability of the sliding-mode control, a control law usually results in large control chattering. In general, there is a trade-off between control chattering and system robustness for the sliding-mode control.

Several model-free approaches based on adaptive control [2, 3], fuzzy control $[4,5]$ and neural network control [6-9] have represented the alternative design methods for the control of uncertain or unknown nonlinear systems. The basic idea of adaptive control is to estimate the plant parameters or controller parameters for the adaptive control design. However, adaptive control can only deal with the systems with known system dynamic structure but unknown system 


\section{Nonlinear systems}

parameters. Fuzzy control using linguistic information is a model-free approach for controlling the dynamic systems. The control rules are fuzzy If-Then rules specifying appropriate control actions. However, it requires the time-consuming trial-and-error tuning procedure for the fuzzy rule design for achieving satisfactory control performance. The key element of neural network control technique is the approximation ability, where the parameterized neural network can approximate the unknown system dynamics or the ideal controller after learning. Although the neural network control can achieve acceptable ontrol performance, the design procedure and computation loading may be overly complex for practical applications.

Recently, some researchers have proposed fuzzy control designs based on the adaptive control scheme. This type of controller is referred to as adaptive fuzzy controllers [10-12]. Based on the universal approximation theorem [10], the adaptive fuzzy control design method can provide a stabilizing controller in the Lyapunov sense. With this approach, the fuzzy rules can be automatically adjusted to achieve satisfactory system response by the adaptive laws. Moreover, some researchers have proposed adaptive fuzzy control designs based on the sliding-mode control scheme. This type of controller is referred to as adaptive fuzzy sliding-mode controller (AFSMC) [13-15]. The AFSMC has the advantages that it can automatically adjust the fuzzy rules like the adaptive fuzzy control and possesses the robustness characteristic against parameter variations and external 
disturbances [13].

In the AFSMC, the adaptive law was designed in the sense of Lyapunov, so that it can guarantee system stability. However, it doesn't consider the modeling error, so that the control performance may be unsatisfied. To solve this problem, this study develops a fuzzy-identification-based adaptive sliding-mode control (FIASMC) scheme to include the modeling-error information into the adaptive laws to achieve better control performance. The FIASMC is composed of a computed controller and a robust controller. The computed controller including a fuzzy identifier is designed to stabilize the system, and the robust controller is used to compensate for the effects of the approximation error. Since the FIASMC utilizes the tracking-error and the modeling-error information simultaneously to derive the adaptive laws, the FIASMC system is superior to the AFSMC for the tracking control performance. In order to illustrate the effectiveness of the proposed FIASMC, it is applied to control a chaotic Duffing system. The performance comparison between the proposed FIASMC and the AFMSC is made.

\section{Fuzzy Approximation System}

There are four principal parts in a fuzzy system: fuzzifier, fuzzy rule base, fuzzy inference engine, and defuzzifier. The fuzzy rule base consists of a collection of fuzzy If-Then rules. Assume that there are $m$ rules in the fuzzy rule 
base and each of them has the following form [4]

$$
\text { Rule } i \text { : If } x_{1} \text { is } F_{1}^{i} \text { and } \ldots x_{n} \text { is } F_{n}^{i} \text { Then } y \text { is } w_{i}
$$

where $x_{j}, j=1,2, \ldots, n$, and $y$ are the input and output variables of the fuzzy system, respectively, and $F_{j}^{i}$ and $w_{i}, i=1,2, \ldots, m$, are the labels of the fuzzy sets. The defuzzification of the output is accomplished by the method of center-of-gravity

$$
y=\sum_{i=1}^{m} \varsigma_{i} \times w_{i} / \sum_{i=1}^{m} \varsigma_{i}
$$

where $\varsigma_{i}$ is the firing weight of the ith rule. If $\varsigma_{i}$ is fixed and $w_{i}$ is viewed as adjustable parameters, equation (2) can be rewritten in the vector form as

$$
y=\mathbf{w}^{T} \xi
$$

where $\mathbf{w}=\left[w_{1}, w_{2}, \ldots, w_{m}\right]^{T} \in R^{m}$ is a parameter vector and $\xi=\left[\xi_{1}, \xi_{2}, \ldots, \xi_{m}\right]^{T}$ $\in R^{m}$ is a regressive vector with $\xi_{i}$ defined as

$$
\xi_{i}=\varsigma_{i} / \sum_{i=1}^{m} \varsigma_{i}
$$

It has been proven that there exists a fuzzy system of (4) such that it can uniformly approximate a nonlinear even time-varying function $\Theta$. By the universal approximation theorem [10], there exists an ideal fuzzy approximator $y^{*}$ such that

$$
\Theta=y^{*}+\varepsilon=\mathbf{w}^{*} \boldsymbol{\xi}+\varepsilon
$$


where $\mathbf{w}^{*}$ is the ideal parameter vector of $\mathbf{w}$, and $\varepsilon$ denotes the approximation error and is assumed to be bounded by $0 \leq|\varepsilon| \leq E$, where $E$ is a positive constant; however, this approximation error bound $E$ is difficult to determine, so that it will be on-line estimated in the following derivation. The approximation error generally decreases as the number of the fuzzy rules $m$ increases. In fact, the ideal parameter vector that is needed to best approximate a given nonlinear function $\Theta$ is also difficult to determine and might not even be unique. Thus, an estimated fuzzy system is defined as

$$
\hat{y}=\hat{\mathbf{w}}^{T} \boldsymbol{\xi}
$$

where $\hat{\mathbf{w}}$ is an estimated parameter vector of $\mathbf{w}^{*}$. Define the estimation error $\tilde{y}$ as

$$
\begin{aligned}
\tilde{y} & =\Theta-\hat{y}=y^{*}-\hat{y}+\varepsilon \\
& =\widetilde{\mathbf{w}}^{T} \xi+\varepsilon
\end{aligned}
$$

where $\tilde{\mathbf{w}}=\mathbf{w}^{*}-\hat{\mathbf{w}}$. To relax the requirement of the uncertain bound $E$ in the practical applications, a bound estimation mechanism is developed to observe the bound of the approximation error. Define the estimation error of the bound

$$
\widetilde{E}=E-\hat{E}
$$

where $\hat{E}$ is the estimated error bound. In the following, the adaptive laws will be derived to on-line tune the estimated parameter vector of the fuzzy system and the bound of approximation error to achieve favorable control performance. 


\section{Ideal Control and Adaptive Fuzzy Sliding-Mode Control}

Consider a class of $n$ th-order nonlinear systems with the controllability canonical form

$$
\begin{aligned}
& \dot{x}_{1}=x_{2} \\
& \dot{x}_{2}=x_{3} \\
& \quad \vdots \\
& \dot{x}_{n}=f\left(x_{1}, x_{2}, \ldots, x_{n}\right)+u
\end{aligned}
$$

or equivalently

$$
x^{(n)}=f(\mathbf{x})+u
$$

where $\mathbf{x}=\left[x, \dot{x}, \ldots, x^{(n-1)}\right]^{T} \in R^{n}$ is the state vector of the system, which is assumed to be available for measurement, $f(\mathbf{x})$ is an unknown real continuous function, and $u$ is the input of the system. The control problem is to find a control law so that the state trajectory $x$ can track a reference trajectory $x_{r}$ closely, where the tracking error is defined as

$$
e=x-x_{r}
$$

and a sliding surface is defined as [16]

$$
s=C(\mathbf{e})-C\left(\mathbf{e}_{0}\right)-\int_{0}^{t} \frac{\partial C(\mathbf{e})}{\partial \mathbf{e}} \mathbf{A}_{\mathbf{m}} \mathbf{e} d \tau
$$

where $\mathbf{e}=\left[e, \dot{e}, \ldots, e^{(n-1)}\right]^{T} \in R^{n}$ is the tracking error vector, $C(\mathbf{e})$ is the vector 
to be designed, $\mathbf{e}_{0}$ is the initial state of $\mathbf{e}$, and $\mathbf{A}_{\mathbf{m}}=\left[\begin{array}{cccc}0 & 1 & 0 & \cdots 0 \\ \vdots & \ddots & \ddots & \ddots 0 \\ 0 & \cdots & 0 & 1 \\ -k_{n} & -k_{n-1} & \cdots & -k_{1}\end{array}\right]$ $\in R^{n \times n}$. If the system dynamics are exactly known, the ideal controller can be designed as [1]

$$
u^{*}=-f(\mathbf{x})+x_{r}^{(n)}-\mathbf{k}^{T} \mathbf{e}-\lambda \operatorname{sgn}(s)
$$

where $\mathbf{k}=\left[k_{n}, \ldots, k_{2}, k_{1}\right]^{T}, \lambda$ is a positive constant and $\operatorname{sgn}(\cdot)$ is the $\operatorname{sign}$ function. Substituting (13) into (10), yields

$$
\dot{\mathbf{e}}=\mathbf{A}_{\mathbf{m}} \mathbf{e}+\mathbf{b}_{\mathrm{m}}[-\lambda \operatorname{sgn}(s)]
$$

where $\mathbf{b}_{\mathbf{m}}=[0, \ldots, 0,1]^{T} \in R^{n}$. Differentiating (12) with respect to time and using the error dynamic equation (14), gives

$$
\dot{s}=\frac{\partial C(\mathbf{e})}{\partial \mathbf{e}} \dot{\mathbf{e}}-\frac{\partial C(\mathbf{e})}{\partial \mathbf{e}} \mathbf{A}_{\mathbf{m}} \mathbf{e}=-\lambda \operatorname{sgn}(s)
$$

where $C(\mathbf{e})$ satisfies $\frac{\partial C(\mathbf{e})}{\partial \mathbf{e}}=[0, \ldots, 0,1]$. In order to drive $s \rightarrow 0$, consider the Lyapunov function candidate as:

$$
V_{1}(s)=\frac{1}{2} s^{2} .
$$

Differentiating (16) with respect to time and using (15), yields

$$
\dot{V}_{1}(s)=s \dot{s}=-\lambda|s| \leq 0 .
$$

In summary, the ideal control system presented in (13) can guarantee the 


\section{Nonlinear systems}

asymptotic stability in the sense of the Lyapunov [1]. In the most cases, the exact model of the nonlinear system dynamics $f(\mathbf{x})$ is always unobtainable; thus the ideal controller $u^{*}$ cannot be implemented. To overcome this drawback, a model-free control technology, which is referred to as adaptive fuzzy sliding-mode control (AFSMC), is proposed first to achieve desired tracking performance. The AFSMC feedback system is shown in Fig. 1, where the controller is assumed to take the following form

$$
u=u_{c}+u_{r}
$$

where the computed controller $u_{c}$ is chosen as

$$
u_{c}=-\hat{f}+x_{r}^{(n)}-\mathbf{k}^{T} \mathbf{e}
$$

with $\hat{f}=\hat{\mathbf{w}}^{T} \boldsymbol{\xi}$ is the fuzzy system for identifying the system dynamic function $f(\mathbf{x})$, and the robust controller $u_{r}$ is designed to compensate for the estimation error between the fuzzy identifier and the system dynamic function. By substituting (18) into (10), yields

$$
\dot{\mathbf{e}}=\mathbf{A}_{\mathbf{m}} \mathbf{e}+\mathbf{b}_{\mathbf{m}}\left(f-\hat{f}+u_{r}\right) .
$$

Differentiating (12) with respect to time and using the error dynamic equation (20), gives

$$
\dot{s}=f-\hat{f}+u_{r}
$$

Then, using the approximation error equation (7), equation (21) can be rewritten as 


$$
\dot{s}=\tilde{\mathbf{w}}^{T} \xi+\varepsilon+u_{r} .
$$

Consider a Lyapunov function in the following form:

$$
V_{2}(s, \tilde{\mathbf{w}}, \tilde{E})=\frac{s^{2}}{2}+\frac{\tilde{\mathbf{w}}^{T} \tilde{\mathbf{w}}}{2 \eta_{1}}+\frac{\tilde{E}^{2}}{2 \eta_{2}}
$$

where $\eta_{1}$ and $\eta_{2}$ are positive constant learning-rates. Differentiating (23) with respect to time and using (22), gives

$$
\begin{aligned}
\dot{V}_{2}(s, \tilde{\mathbf{w}}, \tilde{E}) & =s \dot{s}+\frac{\tilde{\mathbf{w}}^{T} \dot{\tilde{\mathbf{w}}}}{\eta_{1}}+\frac{\tilde{E} \dot{\tilde{E}}}{\eta_{2}} \\
& =s\left(\tilde{\mathbf{w}}^{T} \xi+\varepsilon+u_{r}\right)+\frac{\tilde{\mathbf{w}}^{T} \dot{\tilde{\mathbf{w}}}}{\eta_{1}}+\frac{\tilde{E} \dot{\tilde{E}}}{\eta_{2}} \\
& =\tilde{\mathbf{w}}^{T}\left(s \xi+\frac{\dot{\tilde{\mathbf{w}}}}{\eta_{1}}\right)+s\left(\varepsilon+u_{r}\right)+\frac{\tilde{E} \dot{\tilde{E}}}{\eta_{2}} .
\end{aligned}
$$

If the adaptive laws and the robust controller are chosen as

$$
\begin{aligned}
& \dot{\hat{\mathbf{w}}}=-\dot{\tilde{\mathbf{w}}}=\eta_{1} s \xi \\
& u_{r}=-\hat{E} \operatorname{sgn}(s)
\end{aligned}
$$

where

$$
\dot{\hat{E}}=-\dot{\widetilde{E}}=\eta_{2}|s|
$$

then (24) can be rewritten as

$$
\begin{aligned}
\dot{V}_{2}(s, \tilde{\mathbf{w}}, \tilde{E}) & =\varepsilon s-\hat{E}|s|-(E-\hat{E})|s| \\
& =\varepsilon s-E|s|
\end{aligned}
$$




$$
\leq-(E-|\varepsilon|)|s| \leq 0 .
$$

Since $\dot{V}_{2}(s, \tilde{\mathbf{w}}, \widetilde{E})$ is negative semi-definite, that is $V_{2}(s(t), \tilde{\mathbf{w}}(t), \widetilde{E}(t))$ $\leq V_{2}(s(0), \widetilde{\mathbf{w}}(0), \widetilde{E}(0))$, it implies that $s, \widetilde{\mathbf{w}}$ and $\widetilde{E}$ are bounded. Let function $\Omega \equiv(E-|\varepsilon|) s \leq(E-|\varepsilon|)|s| \leq-\dot{V}_{2}(s, \tilde{\mathbf{w}}, \widetilde{E})$, and integrate $\Omega$ with respect to time, it is obtained that

$$
\int_{0}^{t} \Omega(\tau) d \tau \leq V_{2}(s(0), \tilde{\mathbf{w}}(0), \tilde{E}(0))-V_{2}(s(t), \tilde{\mathbf{w}}(t), \tilde{E}(t))
$$

Because $\quad V_{2}(s(0), \widetilde{\mathbf{w}}(0), \widetilde{E}(0)) \quad$ is bounded, and $\quad V_{2}(s(t), \widetilde{\mathbf{w}}(t), \widetilde{E}(t)) \quad$ is nonincreasing and bounded, the following result can be obtained

$$
\lim _{t \rightarrow \infty} \int_{0}^{t} \Omega(\tau) d \tau<\infty .
$$

Also, since $\dot{\Omega}(t)$ is bounded, by Barbalat's Lemma, it can be shown that $\lim _{t \rightarrow \infty} \Omega=0$ [2]. That is $s(t) \rightarrow 0$ as $t \rightarrow \infty$. As a result, the adaptive fuzzy sliding-mode control system is asymptotically stable.

Although the AFSMC can guarantee system stability; however, it only considers the tracking-error information so that the tracking performance in the adaptive law may be unsatisfied. In the following proposed FIASMC scheme, the modeling-error information will be included in the adaptive laws to achieve better control performance.

\section{Fuzzy-Identification-Based Adaptive Sliding-Mode Control}


The objective of the adaptation mechanism in the adaptive law (25) is to adjust the parameters to drive the tracking error to zero. However, when $|s|$ becomes small, even if the estimated parameter vector $\hat{\mathbf{w}}$ is still far away from its ideal parameter vector $\mathbf{w}^{*}$, the update value of $\mathbf{w}$ in (25) will still be small. This will deteriorate the parameter convergence. In fact, the adaptive law can only detect the parameter error $\tilde{\mathbf{w}}$ via their influence on the tracking error. Thus, it is possible that the parameters do not converge to their idea values, even when the tracking error appears to be small. In this case, the designed controller will be sensitive to noise and operational point charges.

To solve the above problem, an identification model for identifying the nonlinear system (10) is represented as

$$
\begin{aligned}
& \dot{\hat{x}}_{1}=\hat{x}_{2} \\
& \dot{\hat{x}}_{2}=\hat{x}_{3} \\
& \quad \vdots \\
& \dot{\hat{x}}_{n}=\hat{f}(\mathbf{x}, \hat{\mathbf{w}})+u
\end{aligned}
$$

or equivalently

$$
\hat{x}^{(n)}=\hat{f}(\mathbf{x}, \hat{\mathbf{w}})+u
$$

where $\hat{f}(\mathbf{x}, \hat{\mathbf{w}})$ is the fuzzy identifier for on-line estimating the system dynamic function $f(\mathbf{x}), \quad \hat{x}_{i}, i=1,2, \ldots, n$ are the states of the identification model, and $u$ is the input of the system. Adjustment of the fuzzy identifier parameters in the identification scheme can be considered as a process of tuning a set of parameters 
to fit the available input-output data from the system. In the on-line identification scheme, as an input signal excites the controlled system and the fuzzy identifier simultaneously, the modeling error (the difference between the fuzzy identifier output and the system output) is used by an adaptive algorithm for adjusting the model parameters. Define the modeling error as:

$$
\delta=x^{(n)}-\hat{x}^{(n)} .
$$

Using (7), (10) and (32), equation (33) can be rewritten as

$$
\delta=f-\hat{f}=\tilde{\mathbf{w}}^{T} \xi+\varepsilon
$$

It is obvious that the modeling error can be used for adjusting the model parameters and the controller parameters. Therefore, the tracking error and the modeling error can be simultaneously used to tune the controller parameters. In this way, it can intuitively be expected that the derived adaptive law will make the control performance better and the parameter convergence faster and more reliable. Based on the above discussions, the adaptive law utilizing both the tracking-error and the modeling-error information can be derived as

$$
\dot{\hat{\mathbf{w}}}=-\dot{\widetilde{\mathbf{w}}}=\eta_{1}(\eta \delta+s) \xi
$$

where $\eta$ is a positive scaling constant. The overall control scheme of the proposed FIASMC system is shown in Fig. 2. Computing the time derivative of the Lyapunov function in (24) and using (26), (27) and the new adaptive law (35), yields 


$$
\begin{aligned}
\dot{V}_{2}(s, \tilde{\mathbf{w}}, \tilde{E}) & =-\eta \delta \tilde{\mathbf{w}}^{T} \xi+s\left(\varepsilon+u_{r}\right)+\frac{\tilde{E} \dot{\tilde{E}}}{\eta_{2}} \\
& =-\eta \delta(\delta-\varepsilon)+\varepsilon s-\hat{E}|s|-(E-\hat{E})|s| \\
& \leq-\eta \delta(\delta-\varepsilon)-(E-|\varepsilon|)|s| \\
& =-\eta\left[\left(\delta-\frac{\varepsilon}{2}\right)^{2}-\frac{\varepsilon^{2}}{4}\right]-(E-|\varepsilon|)|s| \\
& \approx-\eta\left(\delta-\frac{\varepsilon}{2}\right)^{2}-(E-|\varepsilon|)|s| \\
& \leq-(E-|\varepsilon|)|s| \leq 0
\end{aligned}
$$

where the $\frac{\varepsilon^{2}}{4}$ term is neglected by assuming that it is small enough. Moreover, similar to the discussion below (28), by using the Barbalat's Lemma, it can be concluded that $s(t) \rightarrow 0$ as $t \rightarrow \infty$. As a result, the proposed fuzzy-identification-based adaptive sliding-mode control system is asymptotically stable.

\section{Simulations and Comparisons}

In this section, the proposed FIASMC technique is applied to control a chaotic Duffing system. It should be emphasized that the development of the FIASMC does not need to know the system dynamic function. The chaotic systems have been studied and known to exhibit complex dynamical behavior. 


\section{Nonlinear systems}

The interest in chaotic systems lies mostly upon their complex, unpredictable behavior, and extreme sensitivity to initial conditions as well as parameter variations. Consider a second-order chaotic system such as well know Duffing's equation describing a special nonlinear circuit or a pendulum moving in a viscous medium under control $[17,18]$

$$
\begin{aligned}
\ddot{x}= & -p \dot{x}-p_{1} x-p_{2} x^{3}+q \cos (\omega t)+u \\
& =f(\mathbf{x})+u
\end{aligned}
$$

where $t$ is the time variable; $\omega$ is the frequency; $f(\mathbf{x})=$ $-p \dot{x}-p_{1} x-p_{2} x^{3}+q \cos (\omega t)$ is the system dynamic function; $u$ is the control effort; and $p, p_{1}, p_{2}$ and $q$ are real constants. Depending on the choice of these constants, it is known that the solutions of system (37) may exhibit periodic, almost periodic and chaotic behavior [17]. For observing the chaotic unpredictable behavior, the open-loop system behavior with $u=0$ was simulated with $p=0.4, p_{1}=-1.1, p_{2}=1.0$ and $\omega=1.8$. The phase plane plots from an initial condition point $(0,0)$ are shown in Figs. 3(a) and 3(b) for $q=1.95$ and $q=7.00$, respectively. It is shown that the uncontrolled chaotic dynamic system has different chaotic trajectories for different $q$ values. The control objective is to find a control law so that the chaotic system trajectory can track a desired periodic orbit.

For the practical implementation, a fuzzy system with 5 fuzzy rules is utilized 
to approximate the system dynamic function. The singleton fuzzy rules are initiated from zero and are learned from the derived adaptive laws. The controller parameters are selected as $k_{1}=4, k_{2}=4, \eta=0.5, \eta_{1}=20$, and $\eta_{2}=0.01$. The choice of these parameters is through some trails. The simulation results of the AFSMC for $q=1.95$ and $q=7.00$ are shown in Figs. 4 and 5, respectively. The tracking responses of state $x$ are shown in Figs. 4(a) and 5(a); the tracking responses of state $\dot{x}$ are shown in Figs. 4(b) and 5(b), and the associated control efforts are shown in Figs. 4(c) and 5(c). These simulation results show that the AFSMC design method can achieve the tracking performance; however, the convergence speed is not satisfied. The simulation results of the FIASMC for $q=1.95$ and $q=7.00$ are shown in Figs. 6 and 7, respectively. These simulation results show that the FIASMC can achieve favorable control performance. For evaluation, a tracking index $I$ is defined as $I=\sqrt{e^{2}+\dot{e}^{2}}$. The tracking indexes of AFSMC and FIASMC with $q=1.95$ and $q=7.00$ are shown in Fig. 8. It is shown that the tracking index of the proposed FIASMC is smaller than that of the AFSMC. In summary, since the FIASMC utilizes the tracking-error and the modeling-error information simultaneously, the developed FIASMC scheme can achieve better tracking performance than the AFSMC.

\section{Conclusions}




\section{Nonlinear systems}

This paper proposed a fuzzy-identification-based adaptive sliding-mode control (FIASMC) system. The FIASMC is composed of a computed controller and a robust controller. The computed controller utilizes a fuzzy system to model the system dynamic function, and the robust controller is used to compensate for the effect caused by the approximation error. The proposed FIASMC utilizes both the tracking-error and the model-error information to tune the fuzzy rule, so that it can improve the control performance. The major contributions of this study are (1) the successful integration of the sliding-mode control technique into the adaptive fuzzy controller; (2) the successful development of the FIASMC methodology, which can achieve better performance than the conventional approaches at the expense of a negligible increase in computation loading; (3) the successful application of the proposed technique to control a chaotic system.

\section{Acknowledgment}

The authors appreciate the partial financial support from the National Science Council of Republic of China under grant NSC 90-2213-E-155-016. 


\section{References}

[1] J.J.E. Slotine and W. Li, Applied Nonlinear Control, Englewood Cliffs, NJ: Prentice Hall, 1991.

[2] S. Sastry and M. Bodson, Adaptive Control - Stability, Convergence, and Robustness, Englewood Cliffs, NJ: Prentice Hall, 1989.

[3] P.A. Ioannou and J. Sun, Robust Adaptive Control, Englewood Cliffs, NJ: Prentice Hall, 1996.

[4] J.R. Timothy, Fuzzy Logic with Engineering Application, McGraw-Hill, New York, 1995.

[5] L.X. Wang, A Course in Fuzzy Systems and Control, Prentice-Hall, New Jersey, 1997.

[6] O. Omidvar and D. L. Elliott, Neural Systems for Control, Academic Press, 1997.

[7] C.M. Lin and C.F. Hsu, Neural-network-based adaptive control for induction servomotor drive system, IEEE Trans. Ind. Electron., 49 (2002), 115-123.

[8] C.M. Lin and C.F. Hsu, Neural network hybrid control for antilock braking systems, IEEE Trans. Neural Netw., 14 (2003), 351-359.

[9] J.H. Park, S.H. Huh, S.H. Kim, S.J. Seo, and G.T. Park, Direct adaptive controller for nonaffine nonlinear systems using self-structuring neural networks, IEEE Trans. Neural Netw., 16 (2005), 414-422. 
[10] L.X. Wang, Adaptive Fuzzy Systems and Control: Design and Stability Analysis, Prentice-Hall, Englewood Cliffs, New Jersey, 1994.

[11] S.D. Wang and C.K. Lin, Adaptive tuning of fuzzy controller for robots, Fuzzy Set and Syst., 110 (2000), 351-363.

[12] M. Hojati and S. Gazor, Hybrid adaptive fuzzy identification and control of nonlinear systems, IEEE Trans. Fuzzy Syst., 10 (2002) pp. 198-210.

[13] B. Yoo and W. Ham, Adaptive fuzzy sliding-mode control of nonlinear system, IEEE Trans. Fuzzy Syst., 6 (1998) pp. 315-321.

[14] C.M. Lin and C.F. Hsu, Guidance law design by adaptive fuzzy sliding-mode control, J. Guidance, Contr., Dynamics, 25 (2002), 248-256.

[15] R.J. Wai, C.M. Lin and C.F Hsu, Adaptive fuzzy sliding-mode control for electrical servo drive, Fuzzy Sets and Syst., 143 (2004), 295-310.

[16] R.J. Wai, Total sliding-mode controller for PM synchronous servo motor drive using recurrent fuzzy neural network, IEEE Trans. Ind. Electron., 48 (2001), 926-944.

[17] A. Loria, E. Panteley, and H. Nijmeijer, Control of the chaotic Duffing equation with uncertainty in all parameter, IEEE Trans. Circuits Syst. I, 45 (1998), 1252-1255.

[18] Z.P. Jiang, Advanced feedback control of the chaotic Duffing equation, IEEE Trans. Circuits Syst. I, 49 (2002), 244-249. 


\section{Figure Captions}

Fig. 1. The block diagram of adaptive fuzzy sliding-mode control system.

Fig. 2. The block diagram of fuzzy-identification-based adaptive sliding-mode control system.

Fig. 3. Phase plane of uncontrolled chaotic system.

Fig. 4. Simulation results of AFSMC for $q=1.95$.

Fig. 5. Simulation results of AFSMC for $q=7.00$.

Fig. 6. Simulation results of FISMC for $q=1.95$.

Fig. 7. Simulation results of FISMC for $q=7.00$.

Fig. 8. Comparison of tracking index. 


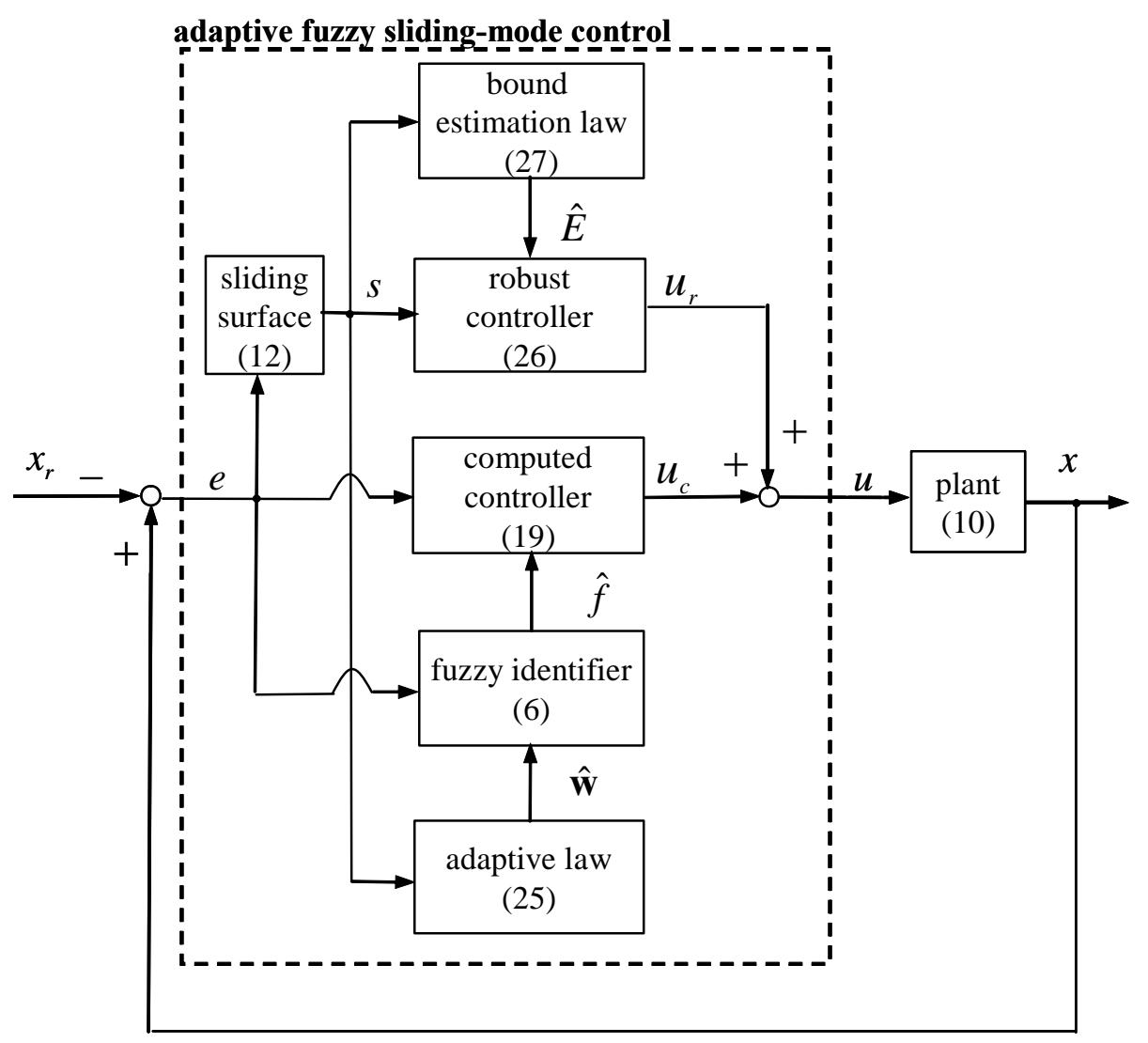

Fig. 1. The block diagram of adaptive fuzzy sliding-mode control system. 


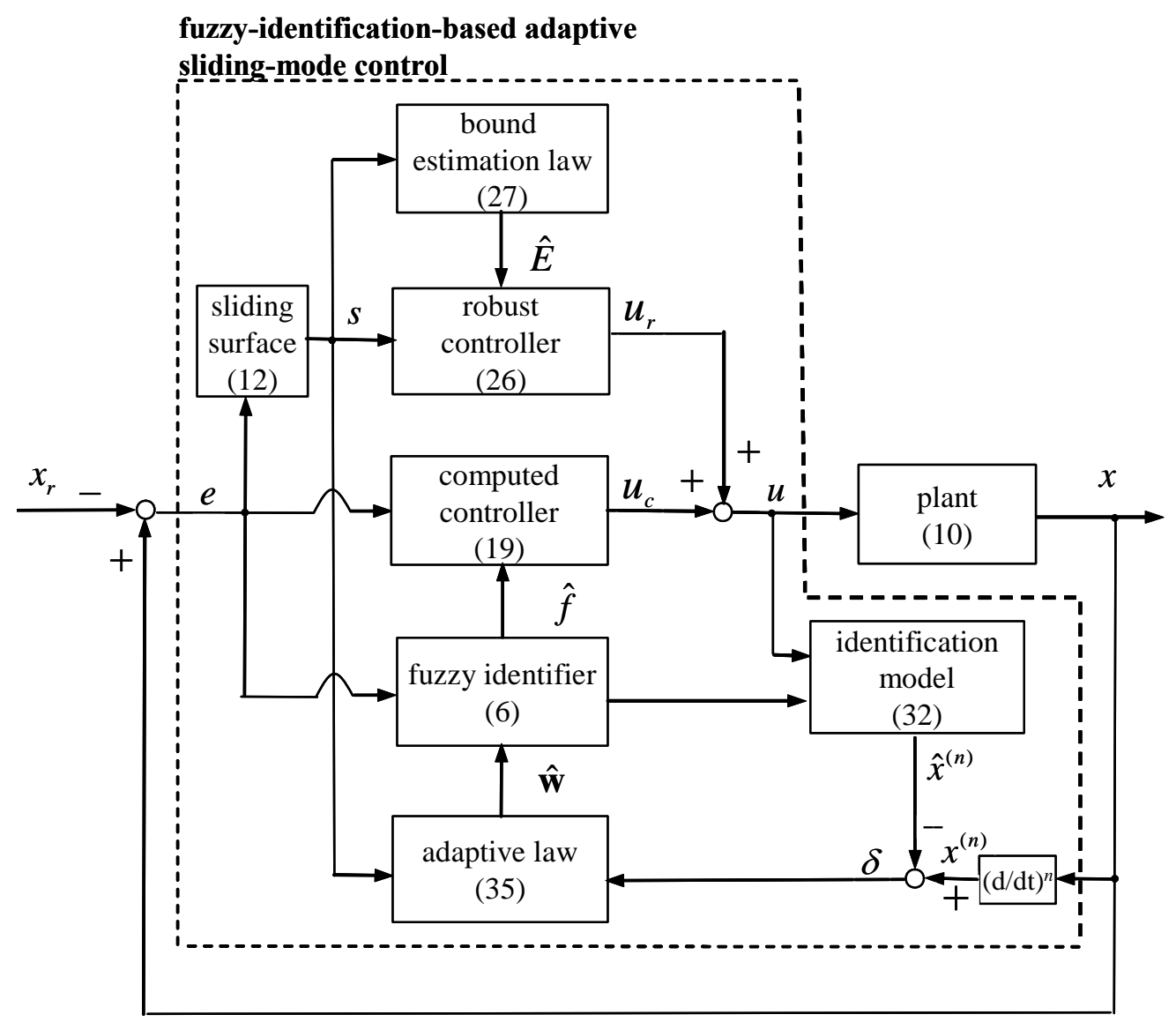

Fig. 2. The block diagram of fuzzy-identification-based adaptive sliding-mode control system. 


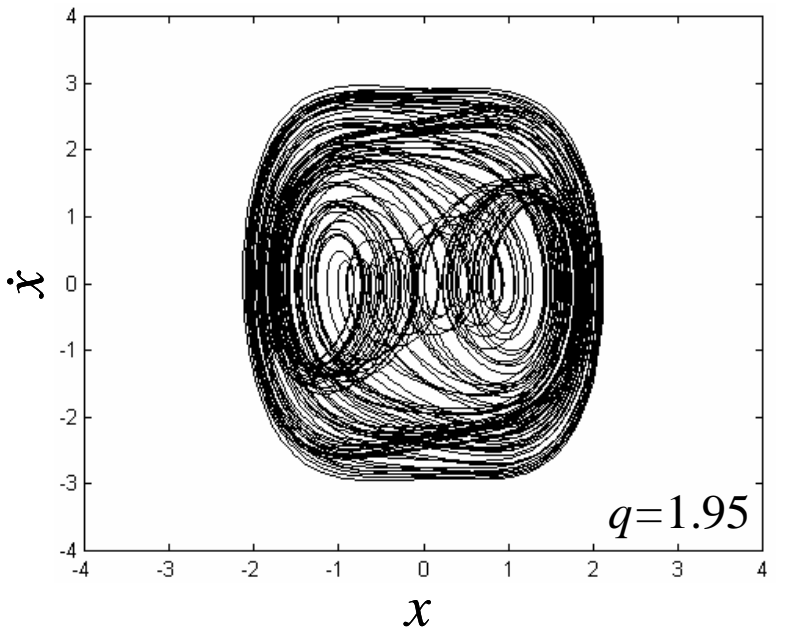

(a)

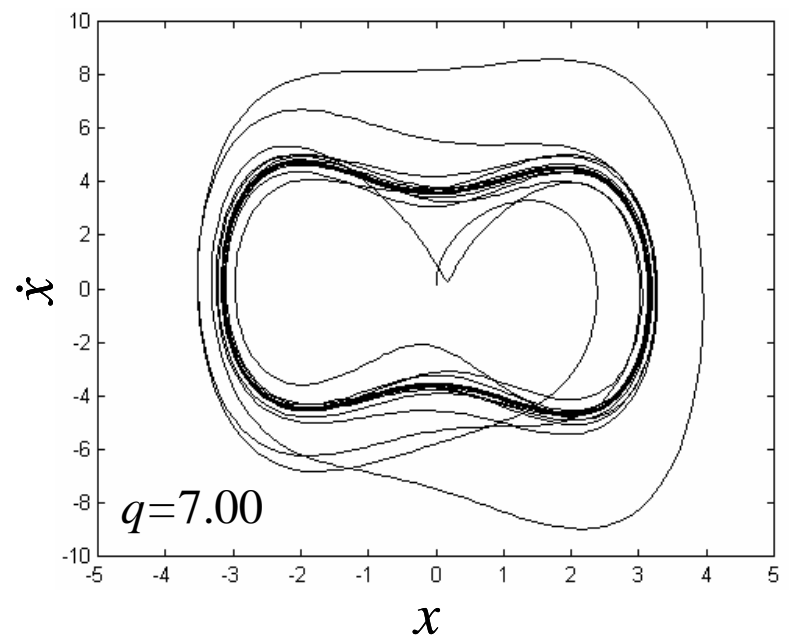

(b)

Fig. 3. Phase plane of uncontrolled chaotic system. 


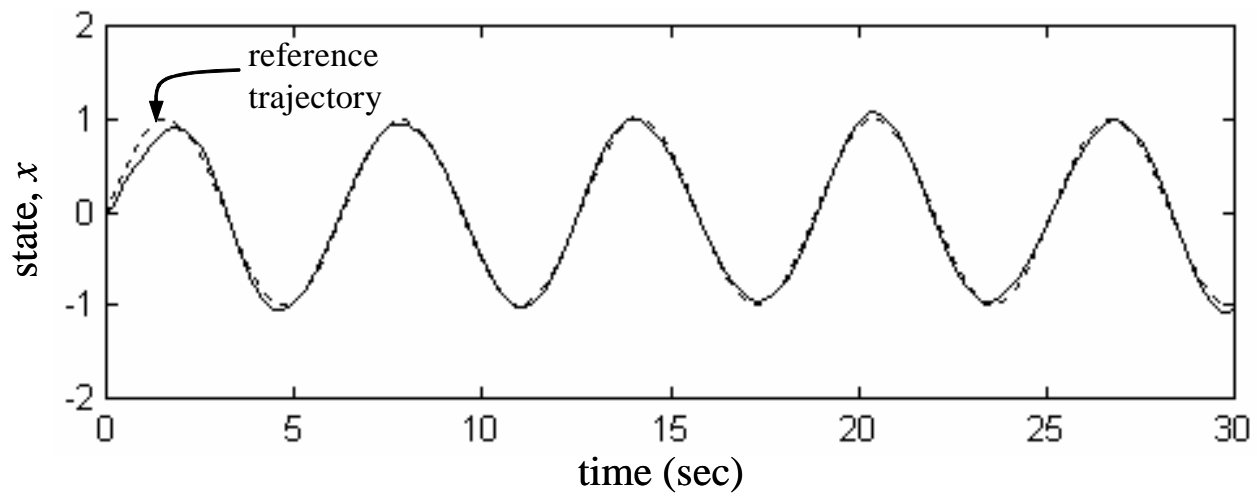

(a)

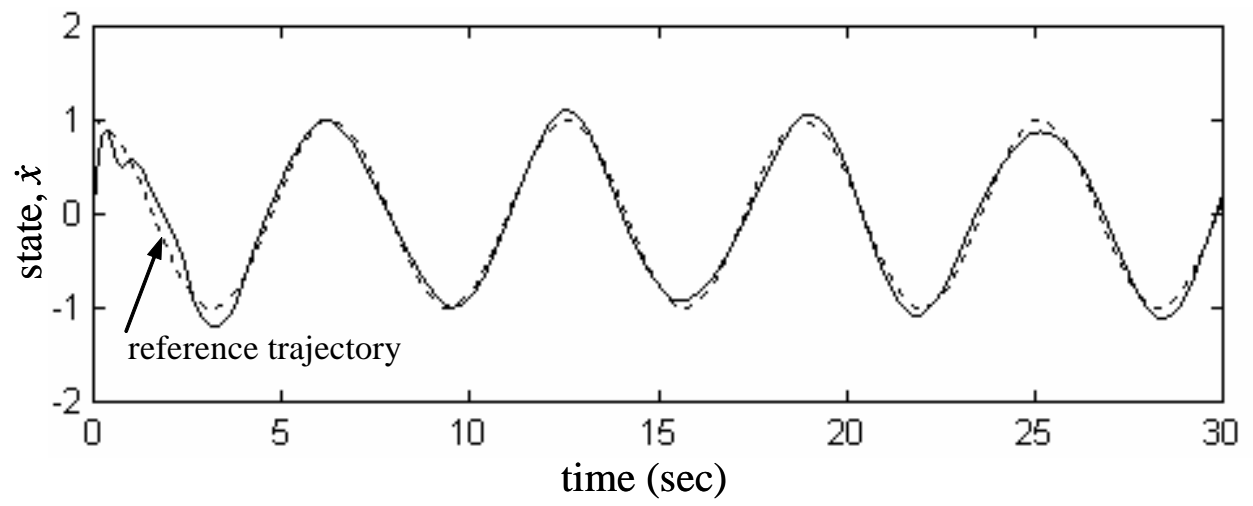

(b)

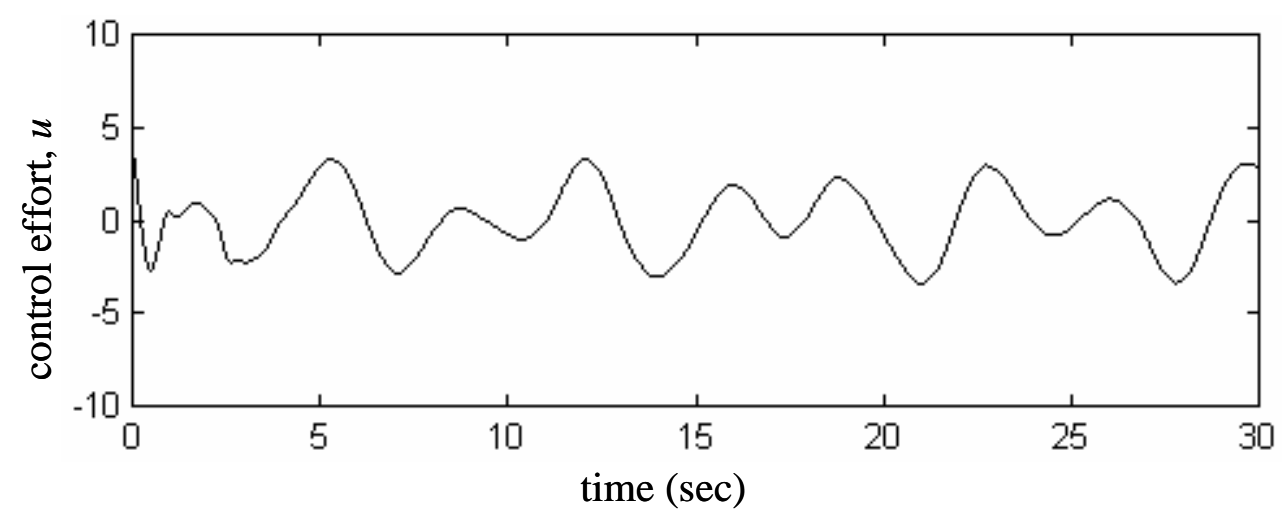

(c)

Fig. 4. Simulation results of AFSMC for $q=1.95$. 


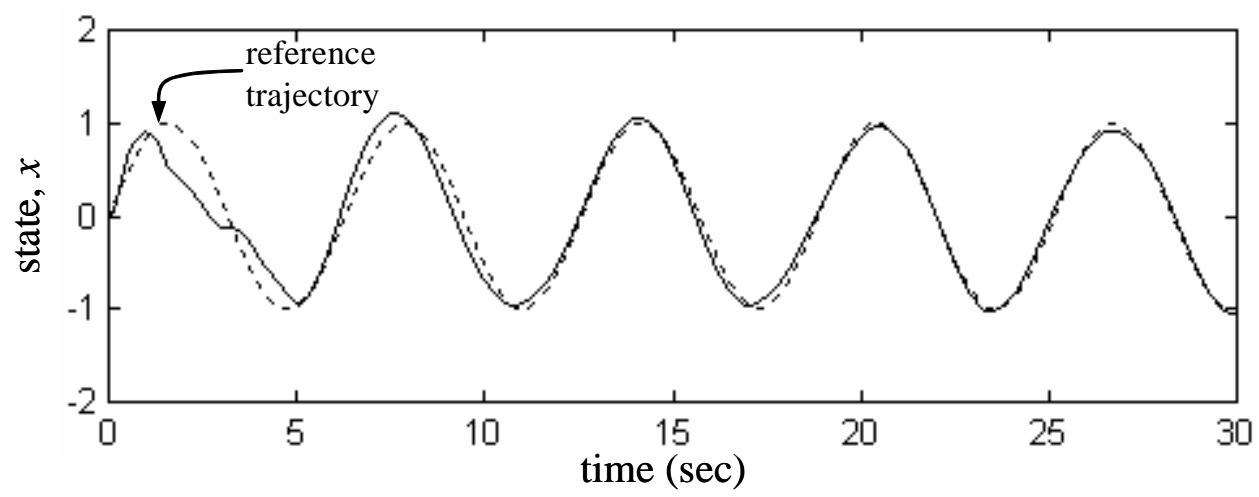

(a)

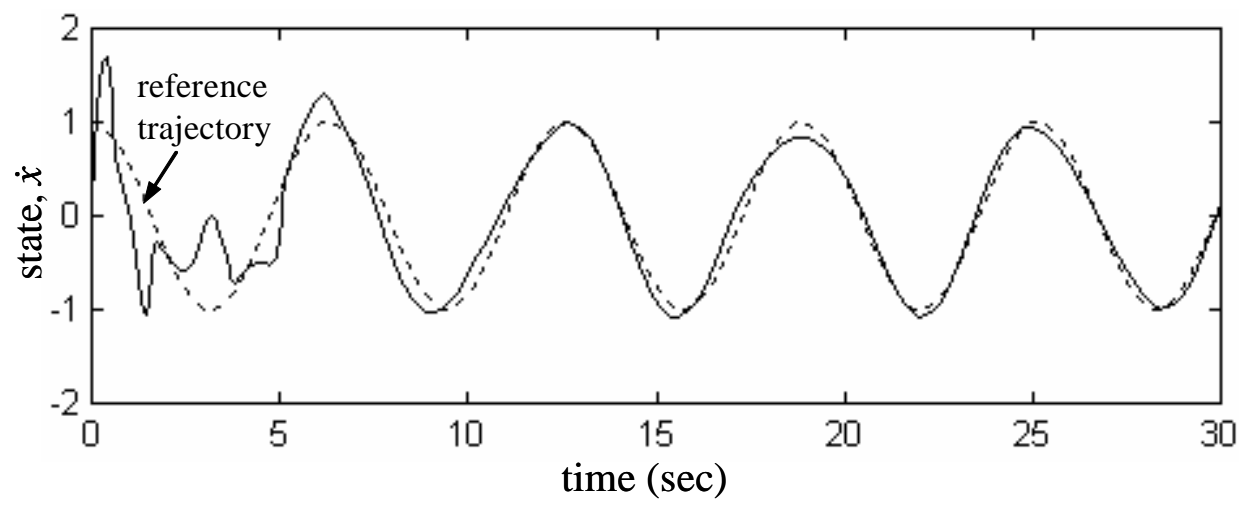

(b)

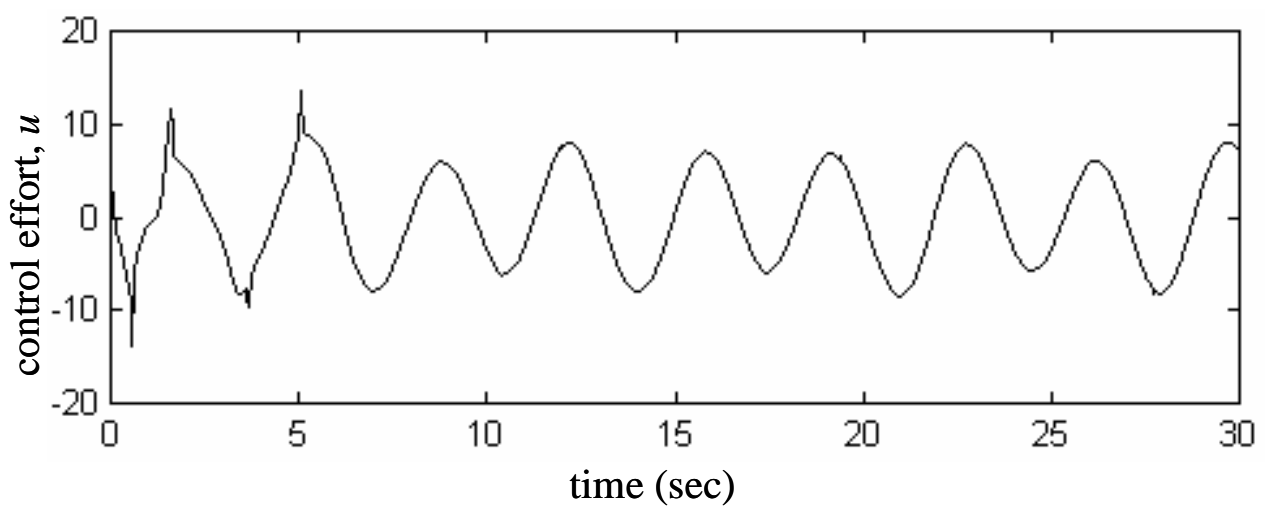

(c)

Fig. 5. Simulation results of AFSMC for $q=7.00$. 


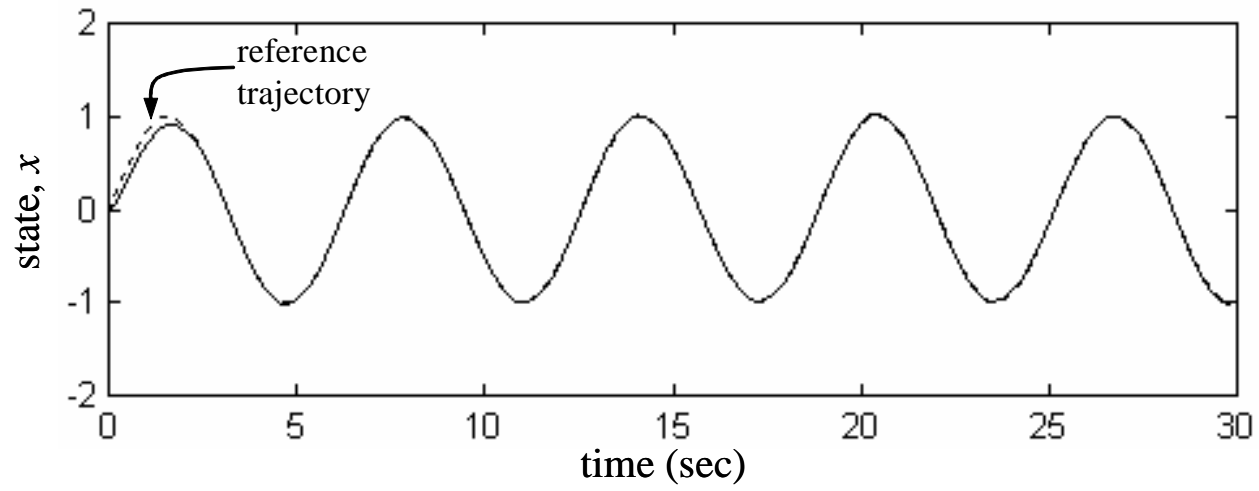

(a)

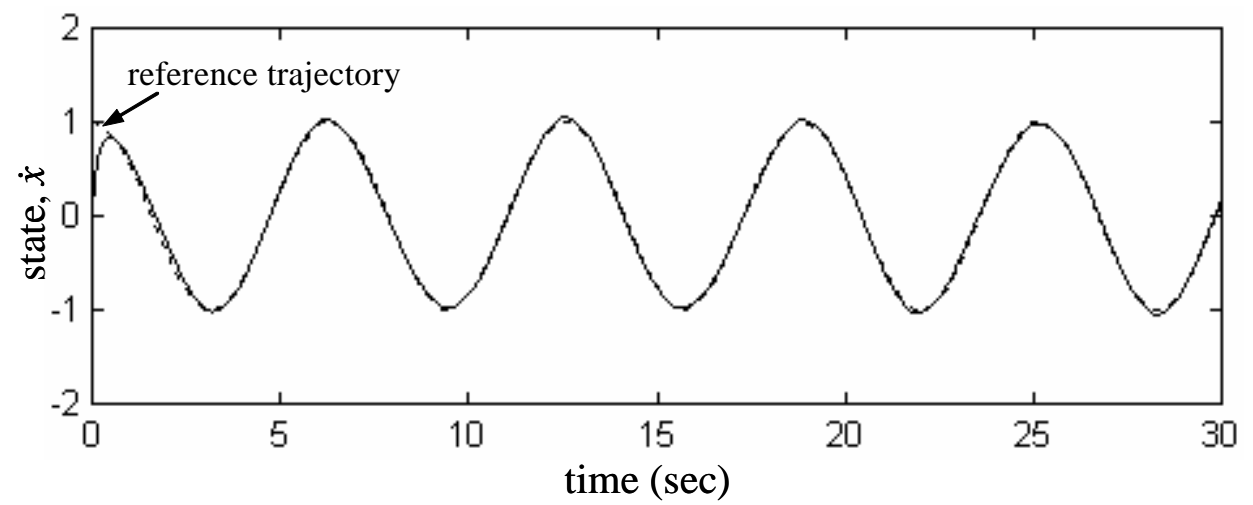

(b)

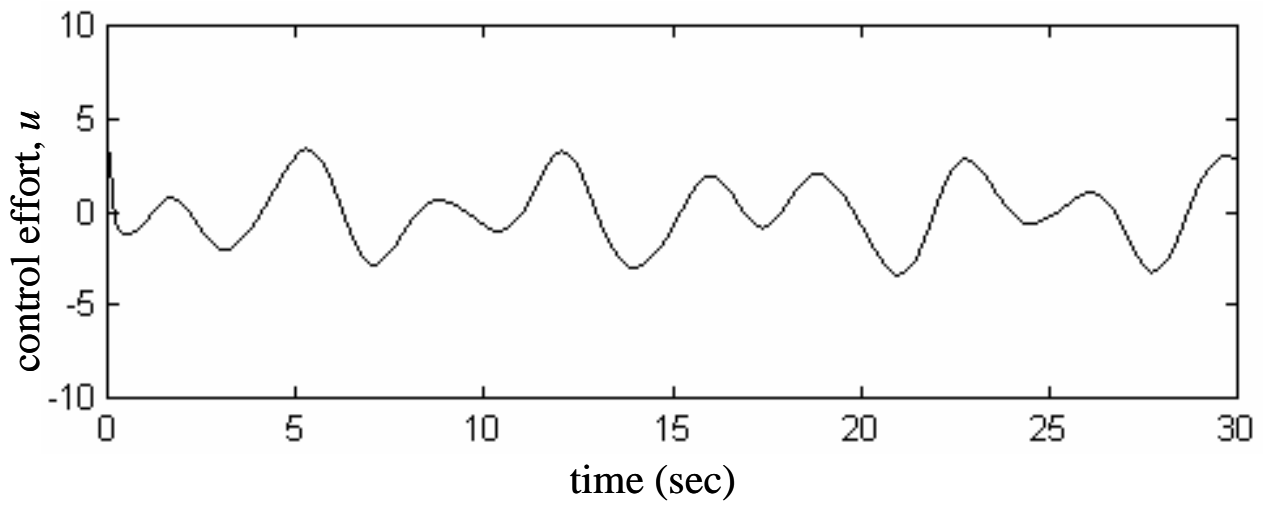

(c)

Fig. 6. Simulation results of FISMC for $q=1.95$. 


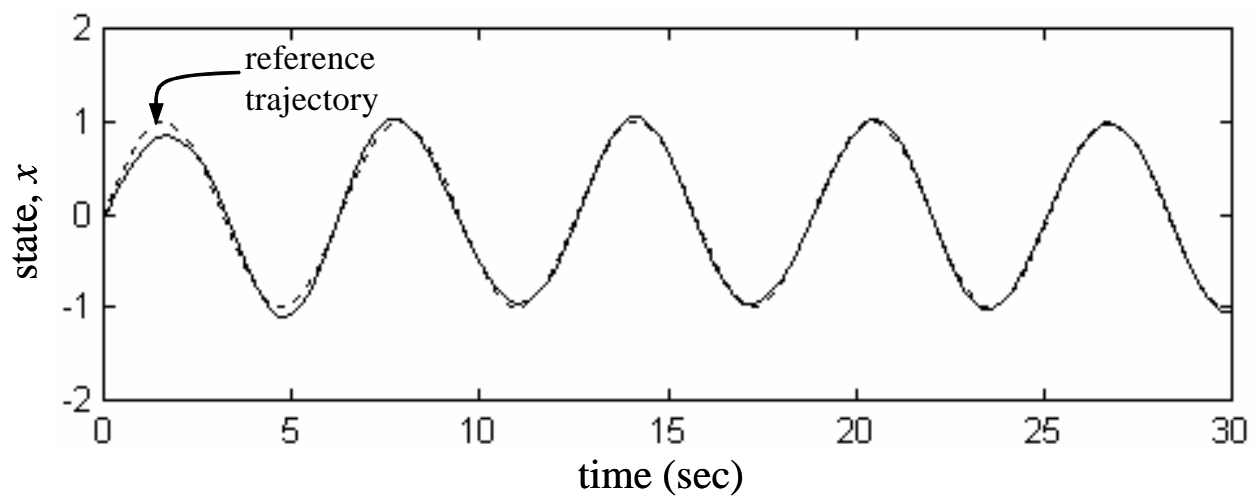

(a)

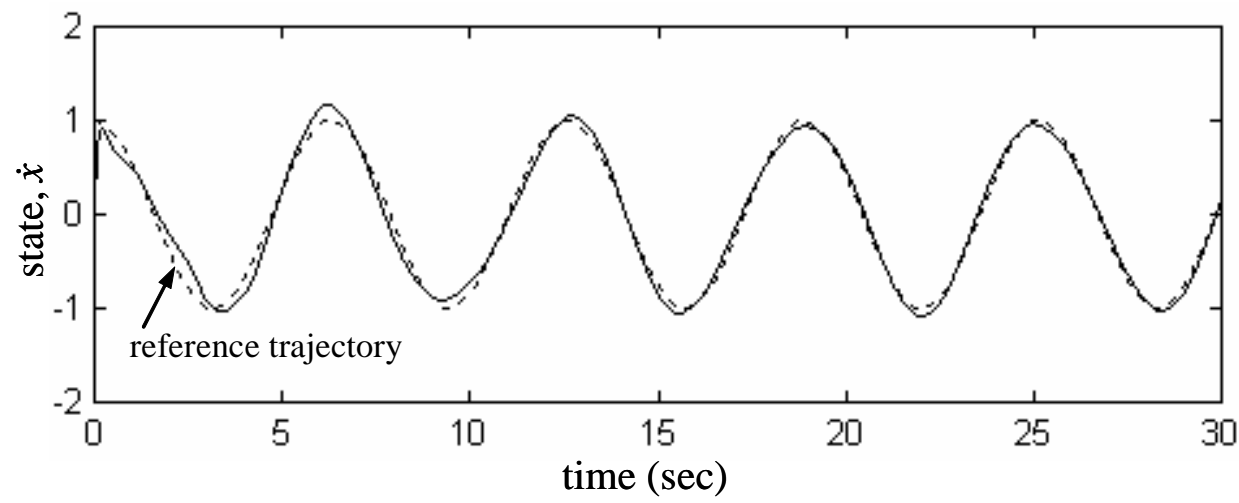

(b)

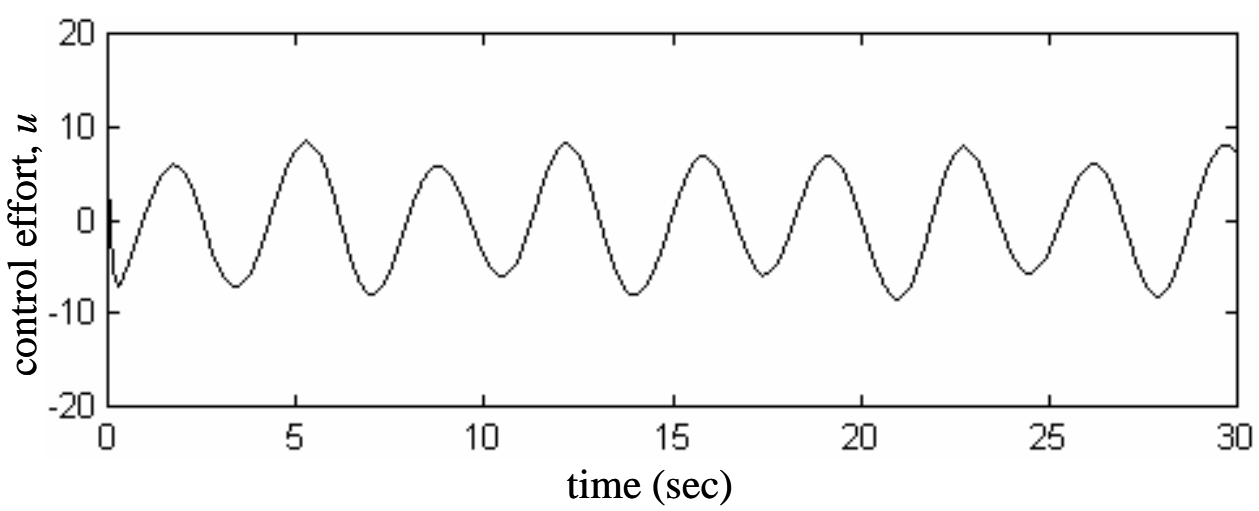

(c)

Fig. 7. Simulation results of FISMC for $q=7.00$. 


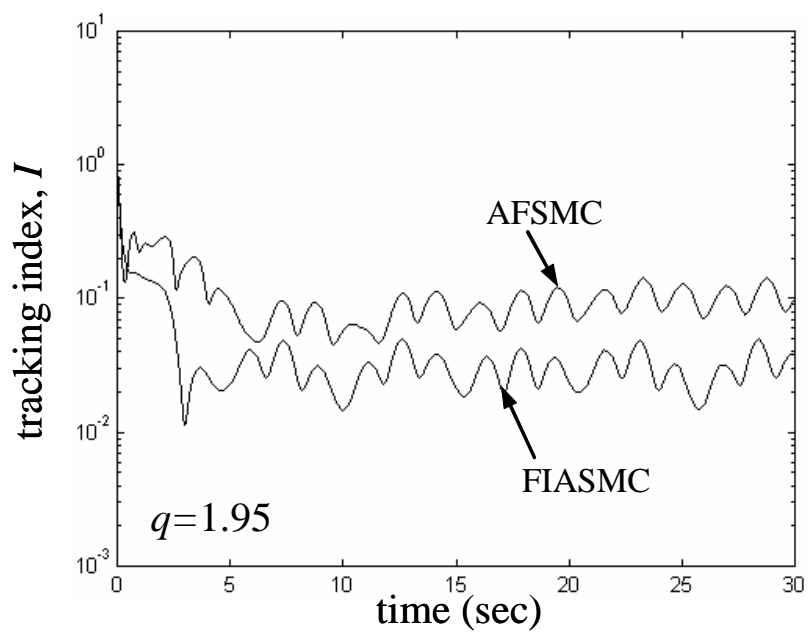

(a)

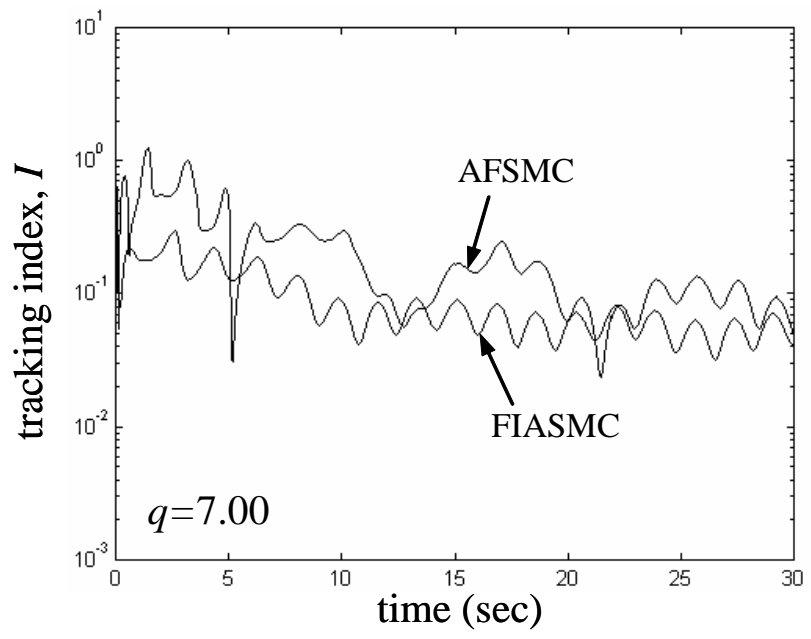

(b)

Fig. 8. Comparison of tracking index.

Received: October 3, 2005 